\title{
MORPHOLOGICAL CHARACTERIZATION OF THE BURSTING AND NONBURSTING NEURONES IN THE OLFACTORY CENTRE OF THE TERRESTRIAL SLUG LIMAX MARGINATUS
}

\author{
SATOSHI WATANABE*, SHIGENORI KAWAHARA AND YUTAKA KIRINO \\ Laboratory of Neurobiophysics, School of Pharmaceutical Sciences, The University of Tokyo, 7-3-1 Hongo, Bunkyo- \\ ku, Tokyo 113-0033, Japan \\ *e-mail: satoshi@mayqueen.f.u-tokyo.ac.jp
}

Accepted 13 January; published on WWW 5 March 1998

\begin{abstract}
Summary
We investigated the morphological characteristics of physiologically characterized neurones in the procerebrum (PC) of the terrestrial slug Limax marginatus. The electrophysiological characteristics of the neurones were determined using the perforated patch recording technique. By comparing the shapes and sizes of somata, we found that the somata of bursting neurones were larger and had more oblong shapes than those of nonbursting

dyes into the bursting and nonbursting neurones revealed morphological differences in the neurites. The bursting neurones had extensive projections within the cell body layer, and the major neurites of the bursting neurones were oriented along the dorsal-ventral axis. In contrast, the nonbursting neurones had neurites extending into the neuropile layers, where terminals of both sensory and presumed output neurones exist.
\end{abstract} neurones, indicating that the bursting and nonbursting neurones in the PC could be discriminated on the basis of the sizes and shapes of their soma. Injection of fluorescent

Key words: procerebrum, Limax marginatus, slug, neural oscillations, fluorescent dye, perforated patch recording.

\section{Introduction}

The procerebrum (PC) of terrestrial pulmonates is a highly developed domain of the cerebral ganglion that is believed to be an olfactory centre because it receives extensive projections from the olfactory nerves (Gelperin et al. 1993) and because its metabolic activities are increased by olfactory stimulation of the tentacle (Chase, 1985). The PC shows highly specialized morphological characteristics (Hanström, 1925; Bullock and Horridge, 1965; Van Mol, 1967; Zs.-Nagy and Sakharov, 1970) and is clearly divided into three layers: the cell, terminal and internal masses. The cell mass consists of densely packed cell bodies, whereas the other masses are neuropile layers where the neurites of both intrinsic and extrinsic neurones occur and possibly make synaptic connections.

The physiological characteristics of the PC were clarified only recently. The PC of Limax marginatus shows a synchronized oscillation of membrane potential (Gelperin and Tank, 1990), like many other olfactory centres in both vertebrates (Adrian, 1942; Freeman, 1978) and invertebrates (Laurent and Naraghi, 1994; Kawahara et al. 1997), although the synchronized activities in L. marginatus are particularly regular and stable. The membrane potential oscillation in the PC of L. marginatus, which is recorded as an oscillation of the local field potential or as optical signals from a potentialsensitive dye, has an average cycle period of approximately 0.7 $\mathrm{Hz}$ and is modulated by olfactory (Gelperin and Tank, 1990;
Delaney et al. 1994; Gervais et al. 1996) and gustatory (Suzuki et al. 1996) stimuli. There is also evidence that the oscillation is related to odour-taste associative learning (Kimura et al. 1996). Optical recording also revealed a prominent spatiotemporal ordering of the activities of neurones in the PC (Kleinfeld et al. 1994; Kawahara et al. 1997). The oscillation has a phase delay along the distal-proximal axis, while the activities along the dorsal-ventral axis are synchronized, resulting in the propagation of waves from distal to proximal regions.

In addition to the macroscopic studies described above, characterization of single PC neurones is essential for elucidating the mechanisms underlying the spatiotemporal order. Recent studies using the perforated patch recording technique have revealed the existence of two types of neurones with different spontaneous activities: bursting neurones, which periodically produce bursts, and nonbursting neurones, which rarely fire (Kleinfeld et al. 1994). These periodic activities of the PC neurones are thought to arise from synaptic interactions including a presumed inhibition of nonbursting neurones by bursting neurones (Kleinfeld et al. 1994). Characterization of the morphological properties of these neurones will give insight into the mechanisms underlying the network activities. The morphology of single PC neurones has been studied in detail in the PC of Helix aspersa (Ratté and Chase, 1997), but 
the relationship between the morphological and physiological characteristics has not been determined. The structure and possibly the physiological properties of the PC of $L$. marginatus are very different from those of $H$. aspersa (Zs.Nagy and Sakharov, 1970; Kawahara et al. 1997). In the present study, we therefore investigated the morphological characteristics of the PC neurones in L. marginatus and their correlation with the electrophysiological properties of the cells. We found that the bursting and nonbursting neurones have distinct morphological characteristics, particularly in the projection patterns of their neurites. Part of this work has been published in abstract form (Watanabe et al. 1997).

\section{Materials and methods}

Limax marginatus from laboratory cultures (weighing $0.8-1.2 \mathrm{~g}$ ) were dissected following anaesthetization by an injection of a $\mathrm{Mg}^{2+}$ buffer into the body cavity. The $\mathrm{Mg}^{2+}$ buffer contained (in mmoll ${ }^{-1}$ ) $\mathrm{MgCl}_{2}, 60$; glucose, 5; and Hepes, 5 (pH 7.6). The cerebral ganglion was isolated in a Petri dish filled with the $\mathrm{Mg}^{2+}$ buffer, and the sheath surrounding the PC was mechanically removed using fine forceps. The preparation was then transferred to a recording chamber and was continuously perfused with a saline solution containing (in mmol ${ }^{-1}$ ) NaCl, 70; KCl, 2; $\mathrm{CaCl}_{2}, 4.9 ; \mathrm{MgCl}_{2}$, 4.7; glucose, 5; and Hepes, 5 (pH 7.6) at approximately $1 \mathrm{ml} \mathrm{min}^{-1}$.

Electrophysiological recording was performed under an upright microscope (Olympus, BX50WI) equipped with a 40× water immersion objective (numerical aperture 0.8 ) and $10 \times$ eyepieces. In some preparations, the largest and smallest diameters of the cell were measured under bright-field illumination, using a micrometer in the eyepiece, before recordings were made. The electrical activity of the neurone was monitored using perforated patch recording (Horn and Marty, 1988; Kleinfeld et al. 1994). The pipette solution contained (in mmoll ${ }^{-1}$ ) potassium gluconate, $70 ; \mathrm{MgCl}_{2}, 5$; Hepes, 5 (pH 7.6); and $100-250 \mu \mathrm{g} \mathrm{ml}^{-1}$ nystatin. The signal was amplified with a patch-clamp amplifier (List Electronic, EPC-7) and recorded on a PCM recorder.

Fluorescent dyes were injected into the PC neurones using a microelectrode after measuring the largest diameter of the neurone. Two dyes were used: sulforhodamine B (Sigma; $1 \%$ aqueous solution) and Lucifer Yellow $\mathrm{CH}$ (Sigma; 3\% aqueous solution). The advantage of using the former was its longer excitation wavelength which evokes little autofluorescence from the ganglia, whereas the latter could be fixed and observed in cleared ganglia. The electrode was pushed into the selected soma with the help of the 'buzz' circuit of the microelectrode amplifier (Nihon Kohden, MEZ-8300). The dyes were injected ionophoretically by applying $1-4 \mathrm{nA}$ negative current pulses ( $1 \mathrm{~Hz}$ and $500 \mathrm{~ms}$ duration) for 2-4 min. Fluorescence was excited using a xenon lamp and mirror units (for sulforhodamine B, Olympus U-MNG; excitation filter $540 \pm 10 \mathrm{~nm}$, dichroic mirror $570 \mathrm{~nm}$, and emission filter $>590 \mathrm{~nm}$; and for Lucifer Yellow, Omega XF14; excitation filter $425 \pm 22.5 \mathrm{~nm}$, dichroic mirror $475 \mathrm{~nm}$, and emission filter
$535 \pm 27.5 \mathrm{~nm})$. The cells were observed either in whole-mount preparations or in sagittal slices (see Fig. 3A). The wholemount preparations were observed either from the posterior or the dorsal surface. Sagittal slices (60-150 $\mu$ m thick) were made with either scalpels or a microslicer after injection of Lucifer Yellow and fixation with phosphate-buffered 10\% formaldehyde. The slices were then dehydrated in ethanol, cleared in methyl salicylate and observed using the same equipment as for the whole-mount preparations.

\section{Results}

The posterior surface of the PC has an almost homogeneous appearance with densely packed cell bodies. However, careful scrutiny revealed that these cells show variations in the size of their cell body (Fig. 1A). Fig. 1B shows the distribution of the largest diameter of the cell bodies in two areas (approximately $0.01 \mathrm{~mm}^{2}$ each) located in the distal and proximal regions, as indicated in Fig. 1C. In the distal area (Fig. 1B1), the distribution was symmetrical and had a peak at $6-7 \mu \mathrm{m}$. In the proximal area (Fig. 1B2), the distribution also had a peak with a similar range $(7-8 \mu \mathrm{m})$ but, in contrast to the distal area, the distribution was asymmetrical with a tail component in the large size ranges, suggesting the existence of a distinct population of large cells.

To relate the physiological properties to the size of the cells, the spontaneous firing patterns of the PC neurones were recorded using the perforated patch recording technique. As reported for $L$. maximus (Kleinfeld et al. 1994), the PC of $L$. marginatus consisted of two types of neurones showing coherent but different types of periodic activity. One type of neurone produced periodic bursts of action potentials (Fig. 2A); the other showed periodic hyperpolarizations and fired at a low frequency between these hyperpolarizations (Fig. 2B). We called the former type a 'bursting neurone' and the latter a 'nonbursting neurone', according to the classification for L. maximus. Of the recorded cells, $87 \%$ (65/75) were unambiguously classified into one or other of these types. The rest of the cells were mostly inactive, possibly due to damage during preparation or to poor recording conditions.

We measured the largest diameter of the soma in the majority of these cells before recording and found that the two types of neurones could be discriminated by the size of their soma. The largest diameter of the soma of the bursting neurone was usually greater than $9 \mu \mathrm{m}$, whereas that of the nonbursting neurone was usually less than $8 \mu \mathrm{m}$ (Fig. 2C). To characterize the shape of the soma further, we also measured the smallest diameter of the somata before recordings were made. The ratio of the largest to the smallest diameter of the bursting neurone was uniformly distributed between 1.0 and 2.0, whereas that of the nonbursting neurones was usually less than 1.2. A combination of the largest diameter and the ratio of the largest to the smallest diameter clearly separated the two types of neurones (Fig. 2D). These findings indicate that the cell bodies of bursting neurones tended to have large oval shapes and those of nonbursting neurones had small globular shapes. In the 

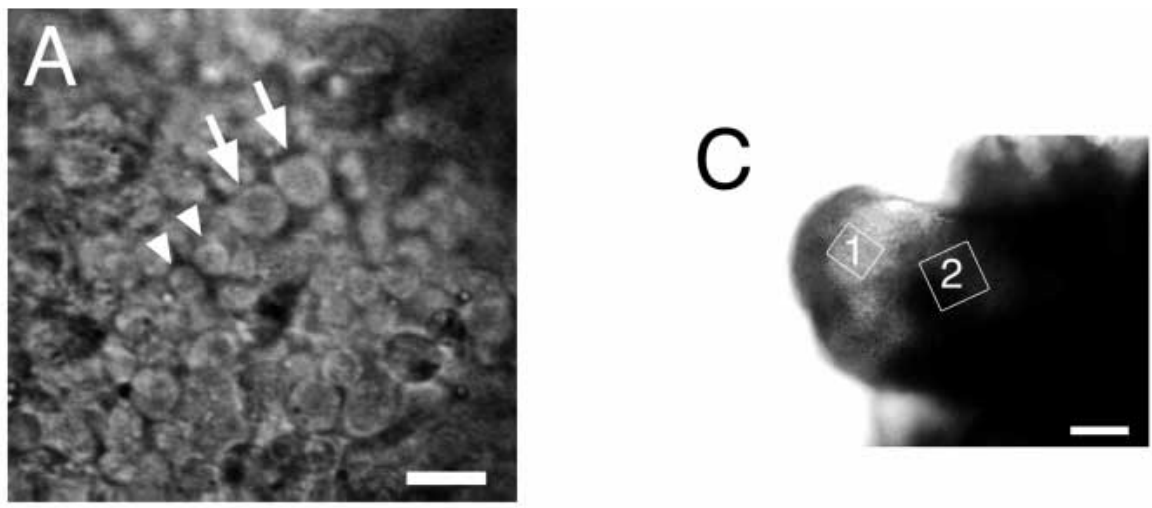

Fig. 1. Variations in the size of the soma of neurones on the posterior surface of the procerebrum (PC). (A) Photograph of the medial surface of the PC. Large neurones (arrows) and small neurones (arrowheads) are shown. Scale bar, $10 \mu \mathrm{m}$. (B) Distribution of the largest diameter of cells in the distal (B1) and the proximal (B2) regions on the surface of the PC. (C) The regions selected for the measurements of the largest diameters in B. 1, the distal region; 2, the proximal region. Scale bar, $100 \mu \mathrm{m}$.

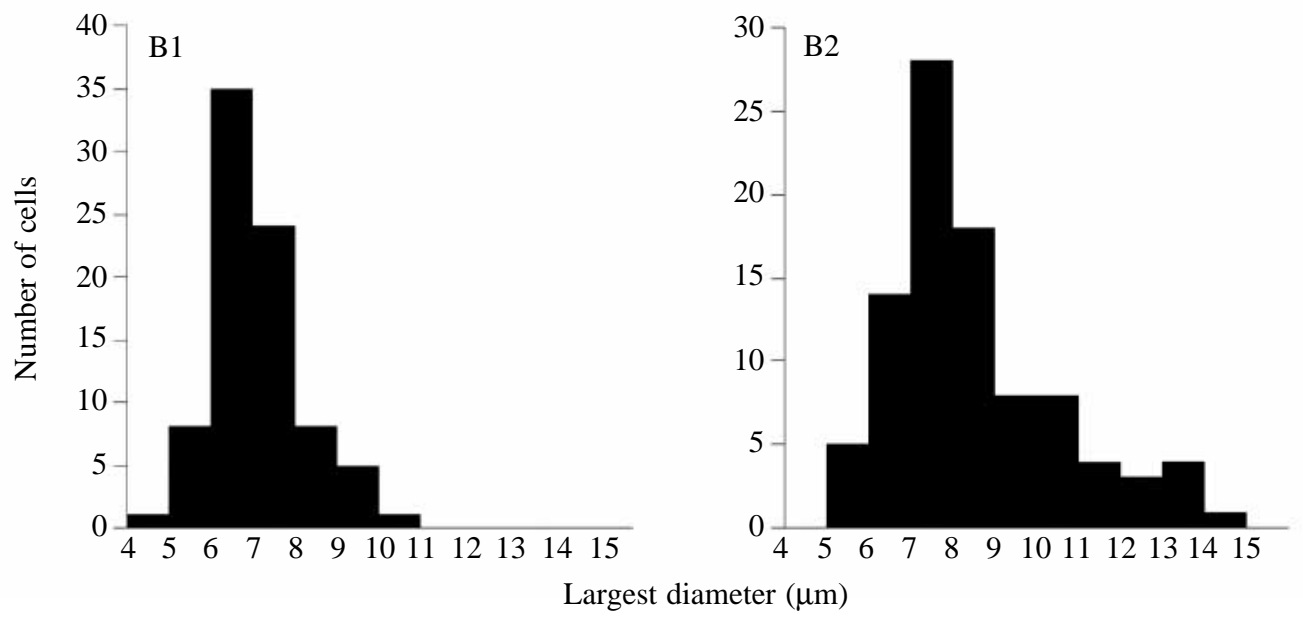

following experiments, we utilized the largest diameter as an operational criterion to determine the type of neurone.

To compare the projection patterns of the neurites of the two types of neurones, fluorescent dye was injected into the soma

after measuring the largest diameter. Using intracellular microelectrodes, we usually observed only small resting potentials and spike amplitudes, but dye injection resulted in clear images of the cell. Observation of the large neurones

Fig. 2. Somatic shapes and electrophysiological properties of the two types of procerebrum (PC) neurones. $(A, B)$ Perforated patch recordings of typical PC neurones in the current-clamp mode. A neurone producing periodic bursts was categorized as a bursting neurone (A), and another neurone firing at a low frequency between periodic hyperpolarizations was categorized as a nonbursting neurone (B). (C,D) Morphological characteristics of the soma of physiologically characterized neurones. The distributions of the largest diameter in bursting and nonbursting neurones were different $(\mathrm{C})$. A plot of the ratio of the largest to the smallest diameter against the largest diameter (D) showed a clear separation between the two types of neurones. Filled circles, bursting neurones; open circles, nonbursting neurones.
A
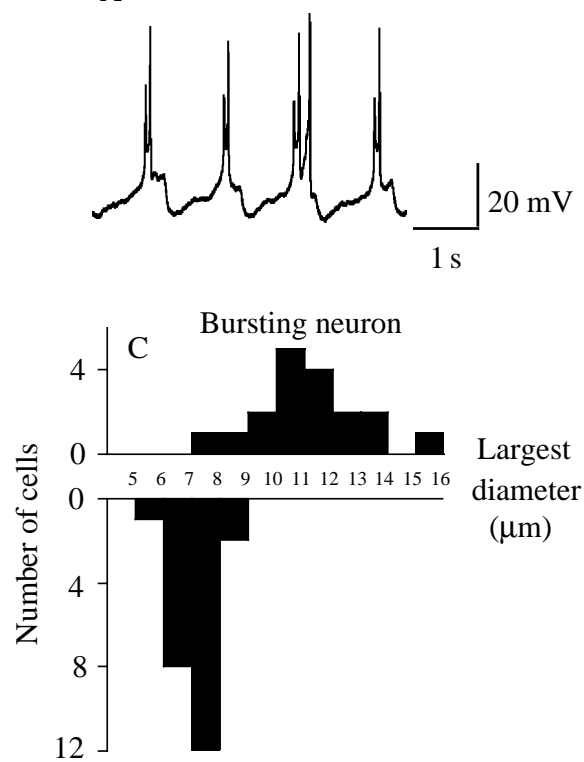

Nonbursting neuron
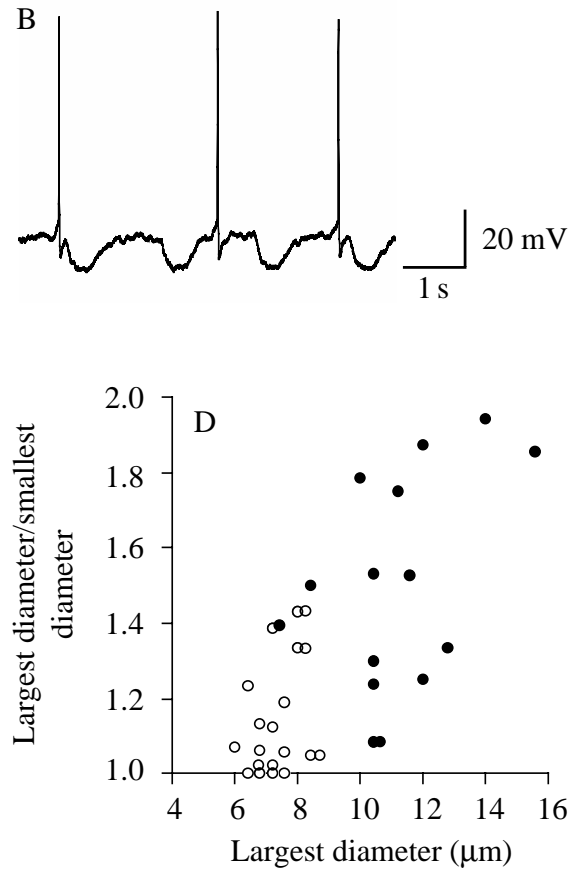
Fig. 3. Morphology of large procerebrum (PC) neurones revealed by the injection of sulforhodamine B or Lucifer Yellow into the soma. (A) Schematic drawing of the PC (left side of the animal). Directions for observation of the whole-mount preparation and the orientation of the sagittal slices are indicated. CM, cell mass; TM, terminal mass; IM, internal mass. (B) Fluorescence photomicrograph (posterior view; right side of the animal) of a large neurone located in the medial region of the PC. A pair of major neurites extends bilaterally along the dorsal-ventral axis. Scale bar, $100 \mu \mathrm{m}$. (C) Tracings of photomicrographs of four large neurones from different preparations (posterior view). The orientation of the PC is the same as in B. Scale bar, $100 \mu \mathrm{m}$. (D) Dorsal view of a large neurone. A pair of neurites (arrowheads) is located near the boundary between the cell mass and the terminal mass. The soma, seen as a large sphere, is out of focus. Scale bar, $50 \mu \mathrm{m}$. (E) Another large neurone in a sagittal section. A neurite (arrowhead) extends horizontally within the cell mass. Scale bar, $25 \mu \mathrm{m}$.

A
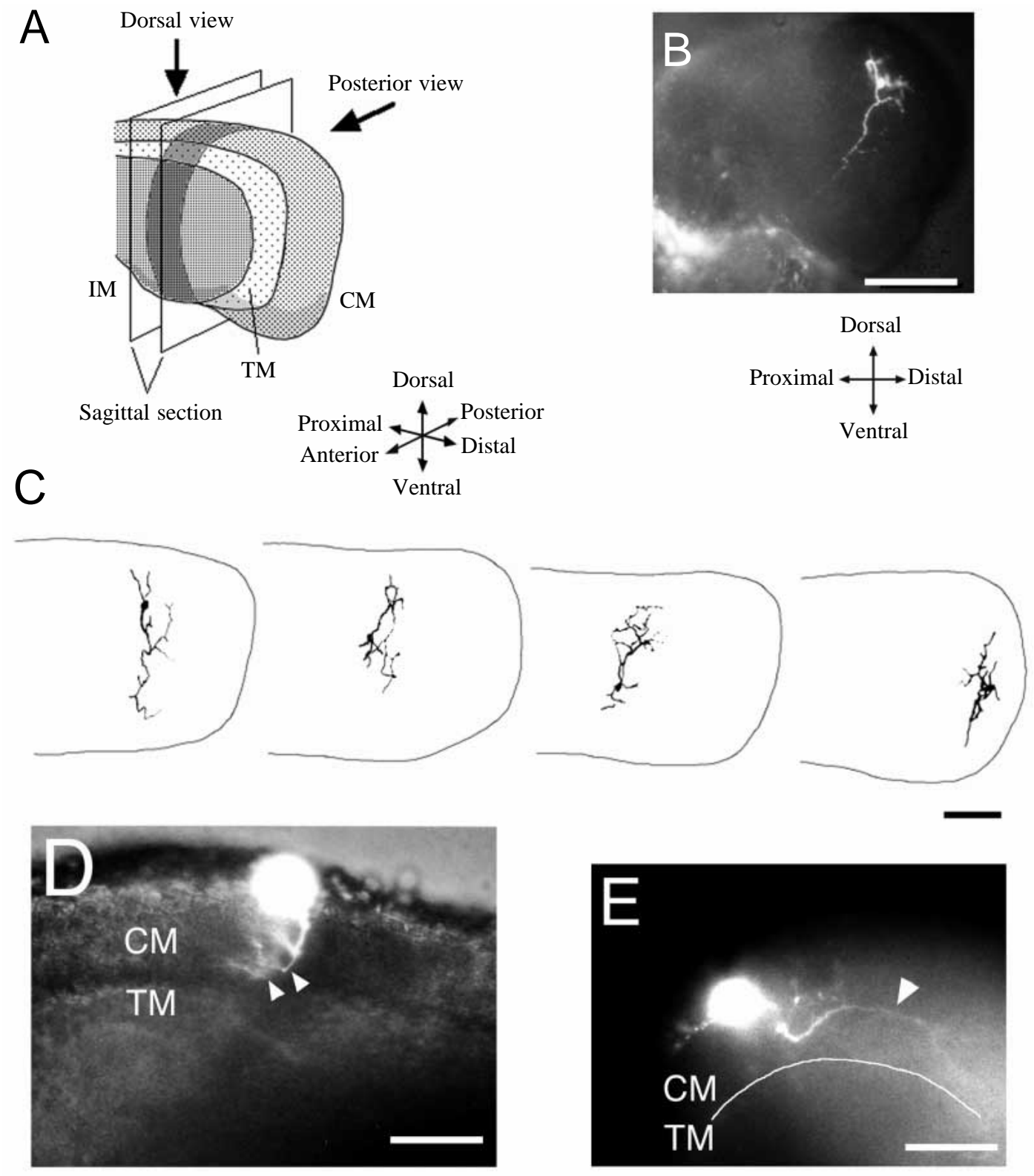

(largest diameter $>9 \mu \mathrm{m}$ ) from the posterior surface revealed that these neurones had 2-4 major neurites which branched further into fine neurites. The neurites of the large neurones had numerous varicosities (data not shown). Interestingly, the major neurites were oriented along the dorsal-ventral axis in 41 cells from a total of 44 injections (27 cells filled with sulforhodamine and 17 with Lucifer Yellow), as shown in Fig. 3B,C. There were, however, two cells giving ambiguous results in which the dominant orientation was not determined and one cell which had a neurite oriented towards the proximal direction. No dominant orientation was observed in the fine neurites. Typically, the main neurites extended bilaterally in both the dorsal and ventral directions, and spanned more than half of the width of the PC along this axis. When observed from the dorsal surface, the large neurones appeared to extend neurites within the cell mass but not into the terminal mass
(Fig. 3D). To eliminate the possibility that the images of the cell mass overlapped with those of the underlying terminal mass, the projections of the neurites of the large neurones were examined in sagittal slices after fixation. These observations of the slices confirmed the previous results: the neurites found in the slices extended horizontally within the cell mass and never appeared in the neuropile layers (Fig. 3E) $(N=10)$.

In contrast to the large neurones, the small neurones (largest diameters $<7 \mu \mathrm{m})$ had a single neurite which descended to the neuropile layers $(N=21 ; 20$ cells filled with sulforhodamine and one with Lucifer Yellow) (Fig. 4). This neurite extended at almost $90^{\circ}$ to the surface of the cell mass and branched extensively in the terminal mass. Some of these branches continued into the internal mass, whereas others apparently ended in the terminal mass. The major neurites entering the internal mass ran parallel to its surface in a distal to proximal 

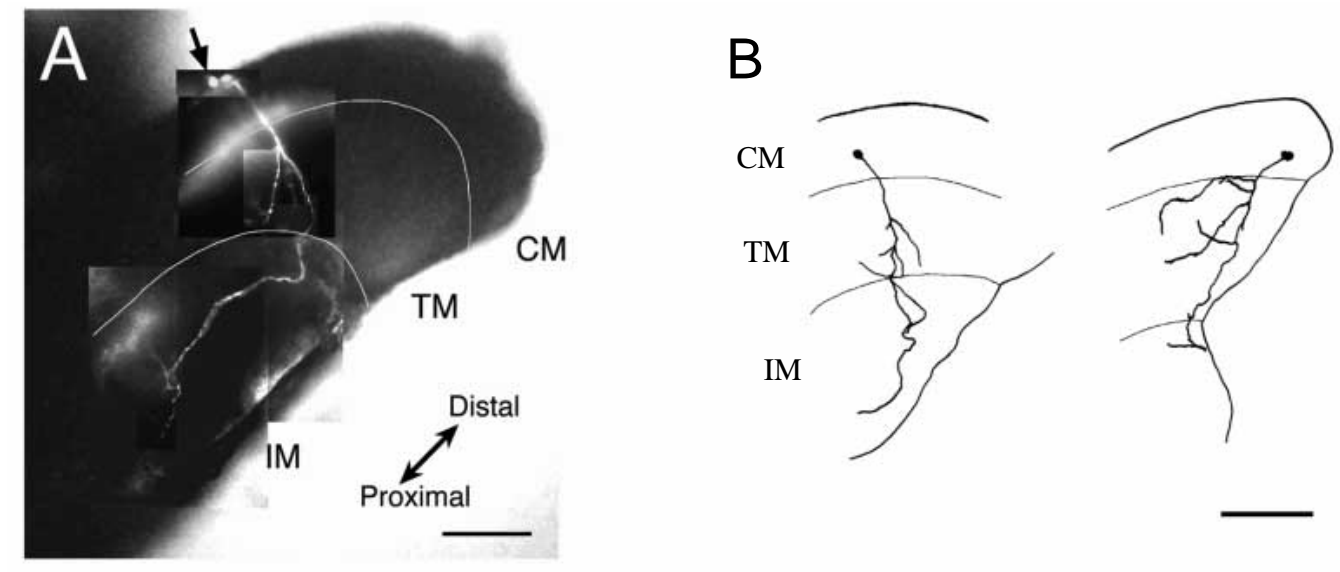

Fig. 4. Morphology of small procerebrum neurones. (A) Sulforhodamine B was injected into the soma (indicated by an arrow), and the preparation was observed from the dorsal surface. A neurite from the soma branched in the terminal mass, and one of the branches extended further into the proximal region of the internal mass. Additional ramifications in the internal mass were also observed. CM, cell mass; TM, terminal mass, IM, internal mass. Scale bar, $50 \mu \mathrm{m}$. (B) Tracings of photomicrographs of two small neurones from different preparations. Scale bar, $50 \mu \mathrm{m}$.

direction. These neurites could usually be traced to the proximal region of the internal mass with some ramifications. Numerous varicosities were also observed in the small neurones, both in the cell mass and in the neuropile layers (data not shown).

\section{Discussion}

In the present study, we showed that PC neurones could be divided into two types on the basis of their distinctive morphological characteristics and that there is a relationship between their morphological and physiological properties. The size distribution of the somata on the surface of the PC (Fig. 1B) implied the existence of morphologically distinct groups of neurones, which is consistent with observations of PC neurones in culture (Rhines et al. 1993). Using the perforated patch recording technique, we showed that the large neurones (largest diameters $>9 \mu \mathrm{m}$ ) corresponded to the bursting neurones and the small neurones (largest diameters $<7 \mu \mathrm{m}$ ) to the nonbursting neurones. Although the diameters in the direction perpendicular to the surface of the ganglion were not determined, our results indicate that measuring the size of the soma from a posterior view is a useful criterion for determining the type of neurone. Since the large neurones correspond to the bursting neurones, the distribution of soma sizes (Fig. 1B) implies an inhomogeneous distribution of bursting neurones, as suggested by optical recording (Kleinfeld et al. 1994).

Injection of fluorescent dye into the soma revealed that the major neurites of the large neurones were oriented along the dorsal-ventral axis. This direction is parallel to that of the synchronization of the propagating waves of the membrane potential revealed by optical recording (Kleinfeld et al. 1994), suggesting that the projection patterns of the bursting neurones are closely related to the spatiotemporal properties of the network activities. Observations from the dorsal surface and in sagittal slices indicated that the major neurites of the large neurones were restricted to the cell mass. This finding suggests that the bursting neurones have local roles, such as producing synchronized oscillations and the propagation of activity. The apparent localization of the neurites of the bursting neurones to the cell mass is consistent with the difference in the temporal patterns of the optical signals between the layers in the PC stained with a potential-sensitive dye (Kawahara et al. 1997). The optical signal from the cell mass, but not from the neuropile layers, showed a rapid depolarizing component, which is likely to be related to the activities of the bursting neurones. The possibility that interactions between bursting neurones are localized is also consistent with the observation that the membrane potential oscillation is generated in a completely isolated cell mass (Gelperin et al. 1993).

In contrast to large neurones, the neurites of small neurones projected to the neuropile layers. This implies that the nonbursting neurones have direct interactions with the olfactory neurones, since the terminal mass is the only projection field of the tentacle nerves within the PC (Kawahara et al. 1997). Similarly, the nonbursting neurones may also transmit signals to output pathways. One such potential pathway is the bundle of nerve fibres projecting to the pedal ganglion found in Achatina fulica (Chase and Tolloczko, 1989). However, we cannot exclude the possibility that bursting neurones also have direct interactions with input or output pathways via unobserved fine neurites. The neurites of some large neurones were found near the border between the cell mass and the terminal mass (data not shown). Direct electrophysiological recording or electron microscopy will be required to determine the synaptic connections between the PC neurones and other pathways.

The existence of morphologically distinct groups of neurones, local and projecting, has also been shown in the olfactory centres of other species. The antennal lobe of the moth Manduca sexta (Waldrop et al. 1987) and the olfactory bulb of mammals (Shepherd, 1972) contain local inhibitory 
interneurones and excitatory projection neurones, suggesting a similar functional organization to that of the olfactory network in L. marginatus. The PC of Helix aspersa has also been shown to contain morphologically different cells, although no formal categorization was possible (Ratté and Chase, 1997).

This work was supported by a JSPS Research Fellowship for Young Scientists (S.W.), a Grant from the Program for Promotion of Basic Research Activities for Innovative Biosciences and Grants-in-Aid (0729105 to Y.K. and 09680646 to S.K.) from the Ministry of Education, Science, Sports and Culture, Japan.

\section{References}

ADRIAN, E. D. (1942). Olfactory reactions in the brain of the hedgehog. J. Physiol., Lond. 100, 459-473.

Bullock, T. H. AND Horridge, G. A. (1965). Structure and Function in the Nervous Systems of Invertebrates, vol. 2. San Francisco: Freeman.

Chase, R. (1985). Responses to odors mapped in snail tentacle and brain by $\left[{ }^{14} \mathrm{C}\right]-2$-deoxyglucose autoradiography. J. Neurosci. 5, 2930-2939.

Chase, R. AND TolloczKo, B. (1989). Interganglionic dendrites constitute an output pathway from the procerebrum of the snail Achatina fulica. J. comp. Neurol. 283, 143-152.

Delaney, K. R., Gelperin, A., Fee, M. S., Flores, J. A., Gervais, R., TANK, D. W. AND KLEINFELD, D. (1994). Waves and stimulusmodulated dynamics in an oscillating olfactory network. Proc. natn. Acad. Sci. U.S.A. 91, 669-673.

FreEman, W. J. (1978). Spatial properties of an EEG event in the olfactory bulb and cortex. Electroencephalogr. clin. Neurophysiol. 44, 586-605.

Gelperin, A., Rhines, L. D., Flores, J. And Tank, D. W. (1993). Coherent network oscillations by olfactory interneurons: modulation by endogenous amines. J. Neurophysiol. 69, 1930-1939.

GelPERIN, A. AND TANK, D. W. (1990). Odour-modulated collective network oscillations of olfactory interneurons in a terrestrial mollusc. Nature 345, 437-440.

Gervais, R., Kleinfeld, D., Delaney, K. R. and Gelperin, A. (1996). Central and reflex neuronal responses elicited by odor in a terrestrial mollusk. J. Neurophysiol. 76, 1327-1339.

HANstRÖM, B. (1925). Über die sogenannten Intelligenzsphären des
Molluskengehirns und die Innervation des Tentakels von Helix. Acta zool. (Stockh.) 6, 183-215.

Horn, R. AND MARTY, A. (1988). Muscarinic activation of ionic currents measured by a new whole-cell recording method. J. gen. Physiol. 92, 145-159.

Kawahara, S., Toda, S., Suzuki, Y., Watanabe, S. and Kirino, Y. (1997). Comparative study on neural oscillation in the procerebrum of the terrestrial slugs Incilaria bilineata and Limax marginatus. J. exp. Biol. 200, 1851-1861.

Kimura, T., Toda, S., Kawahara, S., Sekiguchi, T. and Kirino, Y. (1996). Change of activity pattern induced by learned odors in the olfactory center of a slug. Neurosci. Res. 20, S220.

Kleinfeld, D., Delaney, K. R., Fee, M. S., Flores, J. A., Tank, D. W. AND GELPERIN, A. (1994). Dynamics of propagating waves in the olfactory network of a terrestrial mollusk: an electrical and optical study. J. Neurophysiol. 72, 1402-1419.

LAURENT, G. AND NARAGHI, M. (1994). Odorant-induced oscillations in the mushroom bodies of the locust. J. Neurosci. 14, 2993-3004.

RATTÉ, S. AND ChASE, R. (1997). Morphology of interneurons in the procerebrum of the snail Helix aspersa. J. comp. Neurol. 384, 359-372.

Rhines, L. D., Sokolove, P. G., Flores, J., Tank, D. W. And GELPERIN, A. (1993). Cultured olfactory interneurons from Limax maximus: optical and electrophysiological studies of transmitterevoked responses. J. Neurophysiol. 69, 1940-1947.

SHEPHERD, G. M. (1972). Synaptic organization of the mammalian olfactory bulb. Physiol. Rev. 52, 864-917.

SuzuKi, Y., Kawahara, S., Toda, S. And Kirino, Y. (1996). Effect of gustatory stimulation on the olfactory processing in the CNS of the terrestrial slug. Neurosci. Res. 20, S221.

VAN Mol, J.-J. (1967). Étude morphologique et phylogénétique du ganglion cérébroïde des gastéropodes pulmonés (mollusques). Mém. Acad. R. Belg. (Classe Sci.) 37, 1-168.

Waldrop, B., Christensen, T. A. And Hildebrand, J. G. (1987). GABA-mediated synaptic inhibition of projection neurons in the antennal lobes of the sphinx moth, Manduca sexta. J. comp. Physiol. A 161, 23-32.

Watanabe, S., Kawahara, S. And Kirino, Y. (1997). Morphology and glutamate responses of the procerebral neurons of terrestrial slug Limax marginatus. Neurosci. Res. 21, S229.

Zs.-NAGY, I. AND SAKHAROv, D. A. (1970). The fine structure of the procerebrum of pulmonate molluscs, Helix and Limax. Tissue \& Cell 2, 399-411. 